\title{
Comparison of the Preventive Effects of Epigallocatechin Gallate, Epicatechin Gallate and Epicatechin on Paraquat-Induced Oxidative Stress in Rats
}

\author{
Kiharu IGARASHI, ${ }^{1}$ Orie SUZUKI ${ }^{1}$ and Yukihiko HARA ${ }^{2}$ \\ ${ }^{1}$ Department of Bioproduction, Faculty of Agriculture, Yamagata University, 1-23, Wakaba-machi, Tsuruoka-shi, Yamagata 997- \\ 0037, Japan \\ ${ }^{2}$ Mitui Norin Co. Ltd., 223-1, Miyabara, Fujieda, Shizuoka 426-0133, Japan
}

Received May 20, 1998; Accepted August 11, 1998

\begin{abstract}
The preventative effects of (-)-epigallocatechin gallate (EGCg), (-) -epicatechin gallate (ECg), and (-) -epicatechin (EC) against paraquat-induced oxidative stress were compared in rats in order to discover whether the gallic acid moiety in the EGCg and ECg molecules was necessary to their stronger preventive activity; in addition, the relation between the difference in the chemical structures of their B-ring and their preventive effects was studied. EGCg and ECg strongly suppressed decreases in food intake, body weight gain, and serum and liver triacylglycerol concentrations as well as increases in serum and liver TBARS which were caused by use of the paraquat diet. However, these suppressive effects were almost not observed in EC. The preventive effects of EGCg and ECg against the paraquat-induced oxidative stress were not significantly different. These results indicate that the presence of the gallic acid moiety of EGCg and ECg molecule is important in regard to their stronger preventive effects compared to those of the EC and also that those effects against oxidative stress in the rats were not significantly affected by the differences between the pyrogallol and catechol structures in the B-ring in EGCg and ECg.
\end{abstract}

Keywords: paraquat, catechins, thiobarbituric acid reactive substances, antioxidative enzyme activity

Researchers have studied antioxidative activities of catechins in vitro and it is well-known that $(-)$-epigallocatechin gallate $(\mathrm{EGCg})$ is the strongest catechin, followed by (-) epigallocatechin (EGC), (-) -epicatechin gallate (ECg) and $(-)$-epicatechin (EC). This was determined through the antioxidative activities of catechins in vitro using lard (Hara, 1994). On the other hand, the physiological functions of catechins, e.g. their antimutagenic (Kada et al., 1985; Yen \& Chen, 1995), antitumor (Yoshizawa et al.,1987 ; Susanne et al., 1996), antibacterial (Toda et al., 1991 ; Ikigai et al., 1993), hypocholesterolemic (Fukuyo et al., 1986), and anticomplement (Nakagami et al., 1995) functions have been studied intensively by numerous researchers in relation to the ingestion of large amounts of catechins from food, especially from green tea. However, their preventive effect against oxidative stress in vivo, especially in relation to their chemical structures, has not yet been fully examined. Tsunenari et al. (1992) found that green tea extract and EGCg prolonged the survival rate of mice that had been administered paraquat, which is an agent that produces active oxygens in vivo (Bismuth et al., 1990), and they suggested that EGCg is a potential therapeutic agent for paraquat poisoning that can suppress paraquat-induced oxidative stress. We have previously observed that both EGCg and EGC suppressed paraquat-induced oxidative stress in rats and that the preventative effects of EGCg were stronger than those of EGC (Igarashi et al., 1998).

In this paper, the preventive effects of ECg and EC against oxidative stress, in which the amounts of ECg and EC were smaller than the amount of EGCg in the green tea, were compared in rats to determine whether the preventive effects of ECg and EC differ depending on the presence of gallic acid moiety in the former. In addition, the preventive effects of $\mathrm{EGCg}$ and $\mathrm{ECg}$ against the paraquat-induced oxidative stress were also compared to determine whether the difference in the chemical structure of the B ring of these compounds affects the magnitude of the their preventive effects in regard to oxidative stress.

Although it is known that lungs accumulate paraquat more easily than other organs (Bismuth et al., 1990), the preventive effects of catechins against paraquat-induced oxidative stress were determined in blood and liver in this study, because it has been demonstrated that changes in antioxidative status by administering paraquat was larger in the liver than in the lungs (Igarashi et al., 1998).

\section{Material and Methods}

Catechins and paraquat $\mathrm{EGCg}, \mathrm{ECg}$, and $\mathrm{EC}$ in Fig. 1 were prepared from green tea by the Mitui Norin Co., Ltd. (Fujieda, Shizuoka). The purity level of each compound was more than $95 \%$ when analyzed by HPLC using an ODS column and a mixture of acetonitrile-ethyl acetate- $0.5 \%$ phosphoric acid=12:2:86 (v/v) as a developing solvent. Paraquat (methyl viologen) was purchased from the Sigma Co., Ltd., (St. Louis, Mo).

Animals and diets Four-week-old male Wistar strain rats (Japan SLC, Hamamatsu), each weighing about $50 \mathrm{~g}$, were fed a commercial diet for 5 days before use for the experiment and then divided into 5 groups of 5-6 rats each. Each rat was housed in a stainless-steel cage with a 12-h light 
and 12-h dark cycle (06:00-18:00), at temperature range of $22-$ $24^{\circ} \mathrm{C}$, and a relative humidity of about $55 \%$.

The composition of the experimental diet is given in Table 1. There were five kinds of diets: one was a basal diet without paraquat ( $\mathrm{C}$ diet), and there were four paraquat diets with or without catechins (each, $+\mathrm{PQ},+\mathrm{PQ}+\mathrm{EGCg},+\mathrm{PQ}+\mathrm{ECg}$ and $+\mathrm{PQ}+\mathrm{EC}$ diets). The respective amounts of $0.097 \% \mathrm{ECg}$ and $0.06 \% \mathrm{EC}$ in the $+\mathrm{PQ}+\mathrm{ECg}$ and $+\mathrm{PQ}+\mathrm{EC}$ diets were equal to the amount of $0.1 \% \mathrm{EGCg}$ in the $+\mathrm{PQ}+\mathrm{EGCg}$ diet on a molar basis. EGCg, ECg and EC were added to the + PQ diet instead of a mixture of $\alpha$-corn starch and sucrose (1:1). Food and water were provided ad libitum for 12 days.

Blood and liver. Blood was collected by means of cardiac puncture from rats that had been anesthetized with Nembutal (Dainippon Pharmaceutical Co., Osaka) after $12 \mathrm{~h}$ with no food at the end of the feeding period. A $0.1 \mathrm{ml}$ aliquot of the blood was added to $1.9 \mathrm{ml}$ of physiological saline with gentle shaking, and the mixture was then centrifuged at $1000 \times g$ for $10 \mathrm{~min}$ in order to obtain the serum for measuring the concentration of serum TBARS (thiobarbituric acid reactive substances) as well as to provide erythrocytes. The erythrocytes, which were washed repeatedly with physiological saline and were centrifuged 3 times, were lysed with $\mathrm{H}_{2} \mathrm{O}$ in order to measure their antioxidative enzyme

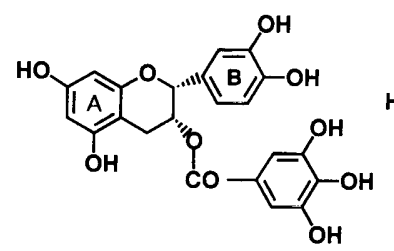

(-)Epicatechin gallate $(\mathrm{ECg})$

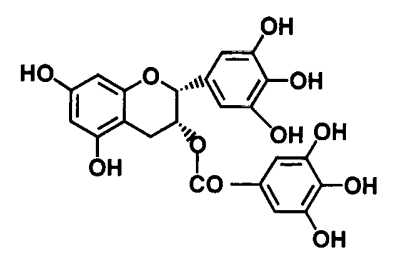

(-)Epigallocatechin gallate (EGCg)

Fig. 1. Chemical structures of catechins. activities. A section of the right lobe of the liver was used to measure the liver superoxide dismutase (SOD), glutathione peroxidase (GSH-Px), catalase, and glutathione reductase (GSSG-R) activities. The other parts of the liver were frozen at $-30^{\circ} \mathrm{C}$ immediately after excision and were stored at $-28^{\circ} \mathrm{C}$ until needed to measure the lipids. The serum used to measure the lipids was prepared by centrifuging the blood at $1000 \times g$ for $15 \mathrm{~min}$.

Preparation of subcellular fractions A supernatant for measuring the antioxidative enzyme activities in liver was prepared according to the method of Del Boccio et al . (1990). One gram of the right lobe was homogenized in $5 \mathrm{ml}$ of ice-cold $0.1 \mathrm{M}$ phosphate buffer $(\mathrm{pH} 7.4)$ containing $1 \mathrm{mM}$ EDTA in a homogenizer with a Teflon pestle, and the homogenate was then mixed with 2 volumes of $2.3 \% \mathrm{KCl}$, followed by centrifugation at $600 \times g$ for $3 \mathrm{~min}$ at $4^{\circ} \mathrm{C}$ in order to remove fibrous materials. The supernatants which were obtained were further centrifuged at $1400 \times g$ for $10 \mathrm{~min}$ at $4^{\circ} \mathrm{C}$ to remove cellular debris. An aliquot of the supernatant was subsequently sonicated at $30 \mathrm{~W}$ for $2 \mathrm{~min}$ ( 4 times for $30 \mathrm{~s}$ ) in a Bransonic model 1200 sonicator (Yamato Co., Tokyo). It was then centrifuged at $10,000 \times g$ for $30 \mathrm{~min}$ at $4^{\circ} \mathrm{C}$ to obtain the mitochondrial fraction needed to measure the SOD and catalase activities. An aliquot of the supernatant was further centrifuged at $105,000 \times \mathrm{g}$ for $60 \mathrm{~min}$ at $4^{\circ} \mathrm{C}$ to obtain the cytosolic fraction needed to measure SOD, catalase, GSH-Px and GSSG-R activities.

Measurements of enzyme activities SOD activity was measured by the xanthine-xanthine oxidase-nitroblue tetrazolium (NBT) system (Imanari et al., 1977), and catalase activity was measured by the spectrophotometric method, following a decrease in absorbance at $240 \mathrm{~nm}$ at $25^{\circ} \mathrm{C}$, due to the decomposition of hydrogen peroxide (Tomita \& Sano, 1983). GSH-Px activity was measured by the procedure of Lawrence and Burk (1976), using $t$-butyl hydroperoxide as a substrate and following the decrease in absorbance of $\mathrm{NADPH}$ at $340 \mathrm{~nm}$ (He \& Yasumoto, 1990). The definitions of one unit of SOD and catalase activities are the amount of enzyme required to inhibit the rate of diformazan formation from NBT by $50 \%$ per mg of hemoglobin or protein and the amount of enzyme needed to decompose $1 \mu \mathrm{mol}$ of $\mathrm{H}_{2} \mathrm{O}_{2}$ in $1 \mathrm{~min}$ per $\mathrm{mg}$ hemoglobin or protein, respectively. The

Table 1. Composition of the diets (\%).

\begin{tabular}{|c|c|c|c|c|c|}
\hline \multirow{2}{*}{ Ingredient } & \multicolumn{5}{|c|}{ Diet } \\
\hline & $\mathrm{C}$ & $+\mathrm{PQ}$ & $+\mathrm{PQ}+\mathrm{EGCg}$ & $+\mathrm{PQ}+\mathrm{ECg}$ & $+\mathrm{PQ}+\mathrm{EC}$ \\
\hline Casein & 20.0 & 20.0 & 20.0 & 20.0 & 20.0 \\
\hline$\alpha$-Corn starch:scurose $(1: 1)$ & 65.5 & 65.48 & 65.38 & 65.383 & 65.42 \\
\hline Cellulose & 5.0 & 5.0 & 5.0 & 5.0 & 5.0 \\
\hline Corn oil & 5.0 & 5.0 & 5.0 & 5.0 & 5.0 \\
\hline Mineral mixture ${ }^{a)}$ & 3.5 & 3.5 & 3.5 & 3.5 & 3.5 \\
\hline Vitamin mixture $^{b)}$ & 1.0 & 1.0 & 1.0 & 1.0 & 1.0 \\
\hline Paraquat & - & 0.02 & 0.02 & 0.02 & 0.02 \\
\hline $\mathrm{EGCg}$ & - & - & 0.1 & - & - \\
\hline $\mathrm{ECg}$ & - & - & - & 0.097 & - \\
\hline $\mathrm{EC}$ & - & - & - & - & 0.06 \\
\hline
\end{tabular}

$\mathrm{C}$, casein diet; $+\mathrm{PQ}$, casein diet with paraquat; $+\mathrm{PQ}+\mathrm{EGCg}$, casein diet with both paraquat and $\mathrm{EGCg} ;+\mathrm{PQ}+\mathrm{ECg}$, casein diet with both paraquat and $\overline{\mathrm{ECg}}$; $+\mathrm{PQ}+\mathrm{EC}$, casein diet with both paraquat and $\mathrm{EC}$. EGCg, $(-)$-epigallocatechin gallate; $\mathrm{ECg},(-)$-epicatechin gallate; EC, (-) -epicatechin.

a) AIN-93G-MX and ${ }^{b)} \mathrm{AIN}-93-\mathrm{VX}$ were obtained from Oriental Co., Tokyo. 
definition of one unit of GSH-Px and GSSG-R activities is the amount of enzyme that is required to oxidize $1 \mu \mathrm{mol}$ of $\mathrm{NADPH}$ in $1 \mathrm{~min}$ per $\mathrm{mg}$ of hemoglobin or protein.

The hemoglobin and protein contents were measured using a commercial hemoglobin test kit (Wako Pure Chemical Ind., Osaka) and using the method of Lowry et al. (1951), respectively.

Measurement of TBARS The concentration of serum TBARS concentration was determined using Yagi's method (1976) and is expressed as nmol of malondialdehyde per $\mathrm{ml}$ of blood. The concentration of liver TBARS was measured using Uchiyama and Mihara's method (1978) using a homogenate which had been prepared using $0.1 \mathrm{M}$ potassium phosphate buffer ( $\mathrm{pH} 7.4)$ which contained $1 \mathrm{mM}$ EDTA.

Lipid analyses The total cholesterol, triacylglycerol, and phospholipid in the serum were measured enzymatically using commercial kits (cholesterol E-test, triglyceride E-test, and phospholipid B-test, respectively; Wako Pure Chemical Ind.). Serum HDL-cholesterol was measured enzymatically in the supernatant that was obtained after heparin-Mn precipitation of the other lipoproteins (Burstein et al., 1970).

The lipids from a frozen liver were extracted and purified by the method of Folch et al. (1957). The amounts of lipids dissolved in isopropyl alcohol after evaporating the chloroform layer containing the purified lipids were measured using the same kits as those used for the serum, except that Mono-test cholesterol (Boehringer Manheim Yamanouchi Co., Tokyo) was used instead of the cholesterol E-test.

Statistical analyses The data for each of the 5 groups were statistically analyzed by Duncan's multiple range test after a one-way analysis of variance (ANOVA) except for the food intake and body weight, which were compared using the Mann-Whitney test for their nonparametric data after 9days. Significant differences in the means were inspected at $p<0.05$.

\section{Results and Discussion}

Food intake and body weight Figure 2 shows the food intake and body weight in the rats fed a control diet and $+P Q$ diets with or without catechins. The supplement of EGCg and ECg in the +PQ diet suppressed decreases in both the food intake and the body weight caused by paraquat. This indicates the possibility that both EGCg and ECg prevented paraquat-induced intoxication, which cause oxidative damage to such organs as the lungs, the liver, the kidneys, and the heart (Barabás et al., 1984; Maktovics et al., 1980). There were no differences in the food intake and body weight gain between the rats that were fed the $+\mathrm{PQ}+\mathrm{EGCg}$ and $+\mathrm{PQ}+$ $\mathrm{ECg}$ diets. This may suggest that the pyrogallol structure in the $\mathrm{B}$ ring of EGCg and the catechol structure in the B ring of $\mathrm{ECg}$ had little effect on their prevention of paraquatinduced oxidative stress. ECg was more effective than EC in regard to suppressing decreases in food intake and body weight, suggesting that the gallic acid moiety in the ECg molecule plays an important role in demonstrating its stronger activity.

Antioxidant status As shown in Table 2, increases in the serum TBARS concentration in the +PQ-rats, when compared with those in the control rats, were significantly suppressed by the supplement of EGCg, ECg, and EC in the $+\mathrm{PQ}$ diet. On the other hand, the increase in the concentration of liver TBARS by the use of $+\mathrm{PQ}$ diet was suppressed by both EGCg and ECg but not by EC.

The erythrocytes SOD, catalase, and GSH-Px activities in the control-, +PQ-, +PQ+EGCg-, $+\mathrm{PQ}+\mathrm{ECg}-$, and $+\mathrm{PQ}+$ EC-rats were not different to a statistically significant degree (Table 2). The activities of SOD, catalase, GSH-Px and GSSG-R in the liver cytosol fractions and the activity of mitochondrial SOD did not show any differences among the control, + PQ-, + PQ + EGCg-, + PQ + ECg-, and +PQ + EC-rats. In contrast, the catalase activity in the mitochondrial fraction decreased markedly in the +PQ-rats compared to that of the control rats. The addition of EGCg and ECg in the $+\mathrm{PQ}$ diet significantly suppressed this decrease; EC, however, did not. These results suggest that the catalase in the mitochondrial fraction may be an enzyme which is sensitive to oxidative stress by paraquat and that the presence of the gallic acid moiety in the EGCg and ECg molecules is very important in regard to suppressing decreases in catalase activity and to express stronger antioxidative activity of $\mathrm{EGCg}$ and $\mathrm{ECg}$ in vivo. Further research as to why there is higher catalase activity in the mitochondrial than in the cytsol fractions is needed, because it is known that the catalase activity in the former is generally weak (Kaur \& Gill, 1986).

Lipid levels As shown in Table 4, the serum total cholesterol decreased significantly in the + PQ- and $+P Q$ + EC-rats, compared to that of the control rats. The supple-
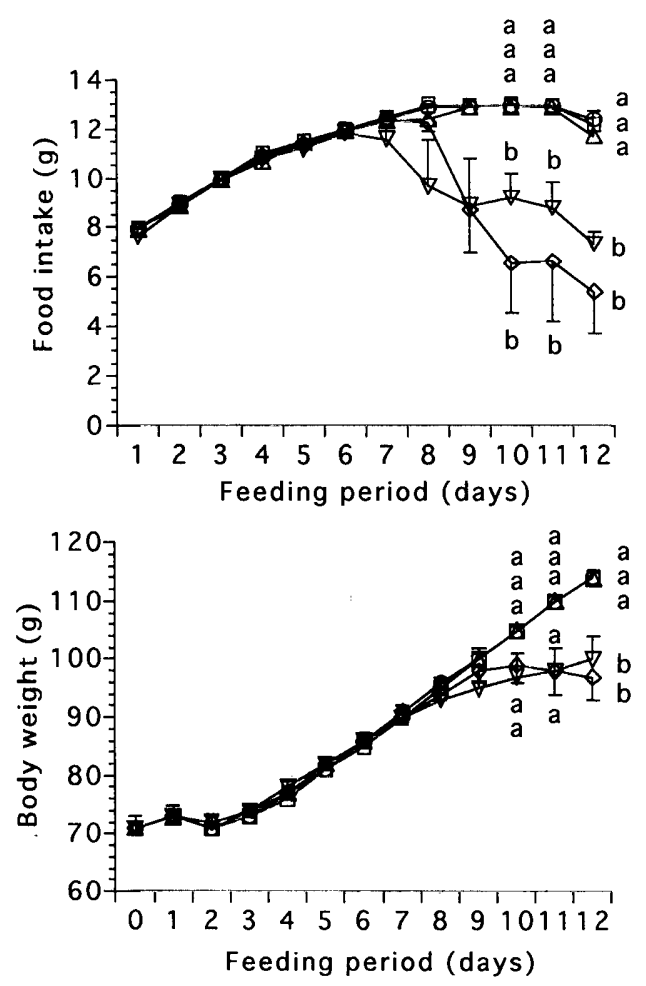

Fig. 2. Comparisons of food intake and body weight of rats fed the paraquat diet that was supplemented with EGCg, ECg and EC. $\square, \mathrm{C}$ diet; $\nabla,+\mathrm{PQ}$ diet; $\triangle,+\mathrm{PQ}+\mathrm{EGCg}$ diet; $\bigcirc,+\mathrm{PQ}+\mathrm{ECg}$ diet; $\diamond, \mathrm{C}+\mathrm{PQ}+\mathrm{EC}$ diet. Each symbol is expressed as mean $\pm \mathrm{SE}$. Symbols that do not share a common letter are significantly different at $p<0.05$ within each indicated day. 
Table 2. Effects of EGCg, ECg and EC on the body weight, liver weight and antioxidant status in rats fed on the paraquat diet.

\begin{tabular}{|c|c|c|c|c|c|}
\hline Group & $\mathrm{C}$ & $+P Q$ & $+\mathrm{PQ}+\mathrm{EGCg}$ & $+\mathrm{PQ}+\mathrm{ECg}$ & $+\mathrm{PQ}+\mathrm{EC}$ \\
\hline Initial body weight $(\mathrm{g})$ & $73 \pm 1$ & $73 \pm 2$ & $73 \pm 1$ & $73 \pm 2$ & $73 \pm 1$ \\
\hline Body weight at 12 days $(\mathrm{g})$ & $114 \pm 1^{a)}$ & $100 \pm 6^{b)}$ & $114 \pm 2^{a)}$ & $114 \pm 2^{a)}$ & $97 \pm 6$ \\
\hline Liver weight $(\mathrm{g} / 100 \mathrm{~g}$ body weight $)$ & $4.25 \pm 0.1^{a)}$ & $3.64 \pm 0.02^{b)}$ & $4.14 \pm 0.1^{a)}$ & $4.26 \pm 0.11^{a)}$ & $3.83 \pm 0.11^{b)}$ \\
\hline Serum TBARS $(\mathrm{nmol} / \mathrm{ml}$ of blood) & $0.86 \pm 0.19^{6)}$ & $2.15 \pm 0.10^{a)}$ & $0.90 \pm 0.18^{b)}$ & $1.05 \pm 0.18^{b)}$ & $1.05 \pm 0.13^{b)}$ \\
\hline Liver TBARS (nmol/ml of blood) & $2.58 \pm 0.06^{b)}$ & $3.85 \pm 0.13^{a)}$ & $2.31 \pm 0.15^{b)}$ & $2.67 \pm 0.17^{b)}$ & $4.06 \pm 0.25^{b)}$ \\
\hline \multicolumn{6}{|c|}{ Antioxidative enzyme activity in erythrocytes } \\
\hline $\mathrm{SOD}(\mathrm{U} / \mathrm{mg}$ of $\mathrm{Hb})$ & $2.51 \pm 0.65$ & $2.59 \pm 0.32$ & $2.87 \pm 0.34$ & $2.81 \pm 0.84$ & $2.45 \pm 0.38$ \\
\hline Catalase (U/mg of $\mathrm{Hb})$ & $151 \pm 21$ & $181 \pm 12$ & $175 \pm 4$ & $180 \pm 25$ & $191 \pm 8$ \\
\hline GSH-Px $(\mathrm{U} / \mathrm{mg}$ of $\mathrm{Hb})$ & $0.09 \pm 0.01$ & $0.10 \pm 0.02$ & $0.12 \pm 002$ & $0.11 \pm 0.02$ & $0.11 \pm 0.01$ \\
\hline \multicolumn{6}{|l|}{ in liver cytosol fraction } \\
\hline SOD $(\mathrm{U} / \mathrm{mg}$ of protein $)$ & $1.42 \pm 0.04$ & $1.45 \pm 0.04$ & $1.41 \pm 0.06$ & $1.41 \pm 0.02$ & $1.40 \pm 0.02$ \\
\hline Catalase $(\mathrm{U} / \mathrm{mg}$ of protein) & $289 \pm 42$ & $283 \pm 27$ & $285 \pm 31$ & $328 \pm 32$ & $386 \pm 55$ \\
\hline GSH-Px (U/mg of protein) & $0.19 \pm 0.02$ & $0.17 \pm 0.01$ & $0.13 \pm 0.02$ & $0.17 \pm 0.02$ & $0.17 \pm 0.01$ \\
\hline GSSG-R(UX10 $10^{-2} / \mathrm{mg}$ of protein) & $0.95 \pm 0.02$ & $1.02 \pm 0.06$ & $1.00 \pm 0.04$ & $0.96 \pm 0.02$ & $0.90 \pm 0.26$ \\
\hline \multicolumn{6}{|l|}{ in liver mitochondrial fraction } \\
\hline $\mathrm{SOD}(\mathrm{U} / \mathrm{mg}$ of protein $)$ & $7.83 \pm 0.42$ & $7.37 \pm 0.35$ & $6.55 \pm 0.30$ & $7.85 \pm 0.5$ & $7.64 \pm 0.32$ \\
\hline Catalase (U/mg of protein) & $1150 \pm 135^{a)}$ & $483 \pm 47^{b)}$ & $1004 \pm 94^{a)}$ & $917 \pm 108^{a)}$ & $483 \pm 63^{b)}$ \\
\hline
\end{tabular}

$\overline{V a l u e s}$ are the mean $\pm \mathrm{SE}$ for $5-6$ rats per group. Values within the same row and not sharing a common superscript are significantly different at $p<0.05$. Values without superscript in the same row are not different statistically.

Table 3. Effects of EGCg, ECg and EC on the serum and liver lipid concentrations.

\begin{tabular}{llllll}
\hline Group & $\mathrm{C}$ & \multicolumn{1}{c}{$+\mathrm{PQ}$} & $+\mathrm{PQ}+\mathrm{EGCg}$ & $+\mathrm{PQ}+\mathrm{ECg}$ & $+\mathrm{PQ}+\mathrm{EC}$ \\
\hline in Serum & & & & \\
$\quad$ Total cholesterol $(\mathrm{mmol} / l)$ & $1.79 \pm 0.06^{a)}$ & $1.59 \pm 0.08^{b)}$ & $1.74 \pm 0.07^{a b)}$ & $1.73 \pm 0.02^{a b)}$ & $1.53 \pm 0.04^{b)}$ \\
$\quad \mathrm{HDL}$ cholesterol $(\mathrm{mmol} / l)$ & $1.57 \pm 0.03$ & $1.42 \pm 0.06$ & $1.50 \pm 0.09$ & $1.55 \pm 0.03$ & $1.41 \pm 0.05$ \\
$\quad$ Triacylglycerol $(\mathrm{mmol} / l)$ & $0.75 \pm 0.09^{a)}$ & $0.36 \pm 0.02^{c)}$ & $0.56 \pm 0.03^{b)}$ & $0.65 \pm 0.07^{a b}$ & $0.33 \pm 0.08^{c)}$ \\
in Liver & & & & & \\
$\quad$ Total cholesterol $(\mu \mathrm{mol} / \mathrm{g}$ of liver $)$ & $5.64 \pm 0.24$ & $5.90 \pm 0.11$ & $5.51 \pm 0.27$ & $5.83 \pm 0.13$ & $5.50 \pm 0.50$ \\
$\quad$ Triacylglycerol $(\mu \mathrm{mol} / \mathrm{g}$ of liver) & $7.01 \pm 0.44^{a)}$ & $3.25 \pm 0.41^{b)}$ & $6.52 \pm 0.40^{a)}$ & $7.07 \pm 0.65^{a)}$ & $2.11 \pm 0.51^{b)}$ \\
$\quad$ Phospholipid & $17.2 \pm 0.5$ & $18.2 \pm 0.2$ & $17.3 \pm 0.4$ & $17.9 \pm 0.5$ & $16.8 \pm 0.6$ \\
\hline
\end{tabular}

Values are the mean \pm SE for 5-6 rats per group. Values within the same row and not sharing a common superscript are significantly different at $p<0.05$. Values without superscript in the same row are not different statistically.

ment of EGCg and ECg in the +PQ diet declined to suppress this decrease. The concentration of serum triacylglycerol decreased significantly in the $+\mathrm{PQ}$-rats compared to that of the control rats. The supplement of EGCg and ECg in the $+\mathrm{PQ}$ diet clearly suppressed this decrease; the supplement of $\mathrm{EC}$ in the $+\mathrm{PQ}$ diet, however, was not effective in this regard.

The liver triacylglycerol concentration in the +PQ-rats showed a marked decrease when compared to that of the control rats, just like the serum triacylglycerol concentration. Supplements of EGCg and ECg in the +PQ diet suppressed these decreases to a statistically significant degree. However, the preventive effects of EC in regard to the decrease in the triacylglycerol concentration were not observed. The decrease in the triacylglycerol concentration in both the serum and the liver of rats fed the $+\mathrm{PQ}$ diet, in comparison to the concentration in the control rats, may have been due to the inhibition of lipid synthesis by paraquat and/or the use of this lipid as an energy source by the rats whose body weight gain was decreasing due to the fall in food intake. The inhibition of lipid synthesis by paraquat may be supported from the report describing that intraperitoneal injection $(50 \mathrm{mg} / \mathrm{kg})$ of paraquat inhibits lipid synthesis (Kornbrust \& Mavis, 1980).

The preventive effects of EGCg and ECg in regard to the decreases in food intake, body weight gain, and the concentrations of serum and liver triacylglycerol as well as decreases in catalase activity in the mitochondrial fraction, in addition, in regard to the increases in serum and liver TBARS, all of which were caused by the use of paraquat diet, were not different at a statistically significant level. On the other hand, the preventive effects of EGCg and ECg against the paraquatinduced oxidative stress were stronger than that of EC. These observations suggest that the gallic acid moiety of EGCg and $\mathrm{ECg}$ is important for preventing the paraquat-induced oxidative stress and that the differences in the chemical structures of the $\mathrm{B}$ ring in the $\mathrm{EGCg}$ and $\mathrm{ECg}$ molecules which have catechol and pyrogallol structures in their B ring, respectively, were not significantly related to their preventative effects.

$\mathrm{ECg}, \mathrm{EGC}$, and EC are known to be absorbed from the digestive tract (Okushiro et al., 1996); therefore, differences in their preventive effects against oxidative stress in vivo may be related in part to the differences in the antioxidative activities of those compounds which were absorbed. However, because it is reported that recovery of the administered EGCg in the blood is low (Nakagawa \& Miyazawa, 1997), it may be necessary to investigate the metabolites of catechins and further to determine the relation between the chemical structures of metabolites and their preventive effects against oxidative stress to elucidate the differences in the preventive activities of catechins in vivo. 
Acknowledgments This work was supported in part by a Grant-inAid for Scientific Research (Project No. 09556025) from the Ministry of Education and Culture of Japan.

\section{References}

Barabás, K., Szabó, L., Matkovics, B. and Berencsi, G. (1984). Effects of paraquat on peroxide metabolism enzymes and lipid peroxidation in the rats. Gen. Pharmacol., 15, 133-137.

Bismuth, C., Garnier, R., Baud, F.J., Muszynski, J. and Keyes, C. (1990). Paraquat poisoning, an overview of the current status. Drug Saf., 5, 243-251.

Burstein, M., Scholnick, H.R. and Morfin, R. (1970). Rapid method for the isolation of lipoproteins from human serum by precipitation with polyanions. J. Lipid Res., 11, 583-595.

Del Boccio, G., Lapenna, D., Porreca, E., Pannelli, A., Savini, F., Feliciani, P., Ricci, G. and Cuccurulle, F.(1990). Aortic antioxidan defense mechanism: Time-related changes in cholesterol-fed rabbits. Atherosclerosis, 81, 127-135.

Folch, J., Lees, M. and Sloane-Stanley, G.H. (1957). A simple method for the isolation and purification of total lipids from animal tissues J. Biol. Chem., 226, 497-509.

Fukuyo, M., Hara, Y. and Muramatsu, K. (1986). Effects of tea leaf catechin, (-)-epigallocatechin gallate, on plasma cholesterol level in rats. Nippon Eiyo Shokuryo Gakkaishi, 39, 495-500 (in Japanese).

Hara, Y. (1994). Prophylactic functions of tea polyphenols. In "Food Phytochemicals for Cancer Prevention II," ed. by C-T. Ho, T. Osawa, M.-T. Huang and R.T. Rosen, American Chemical Society, 547, Washington, DC, pp. 34-50.

He, P. and Yasumoto, K. (1990). Age-associated changes in glutathione peroxidase and oxidized protein in erythrocytes of senescenceaccelerated mice. Nippon Eiyo Shokuryo Gakkaishi, 43, 121-125 (in Japanese)

Ikigai, H., Nakano, T., Hara, Y. and Shimamura, T. (1993). Bacteriocidal catechins damages the lipid bilayer. Biochim. Biophys. Acta, 1147, 132-136.

Igarashi, K., Suzuki, O., Hara. Y., Yoshiki, Y. and Okubo, K. (1998). Comparison of the protective effects of epigallocatechin gallate and epigallocatechin on paraquat-induced oxidative stress in rats. Food Sci. Technol. Int. Tokyo, 4, 149-154.

Imanari, T., Hirata, M., Miyazaki, M. and Hayakawa, K. (1977). Improved assay method for superoxide dismutase. Igaku no Ayumi, 101, 496-497 (in Japanese)

Kada, T., Kaneko, K., Matsuzaki, S., Matsuzaki, T. and Hara, Y. (1985). Detection and chemical identification of natural bio-antimutagens, a case of the green tea factor. Nutr. Res., 150, 127-135.

Kaur, S. and Gill, S.S. (1986). Distribution and nature of epoxide hydrolase activity in subcellular organelles of mouse liver. Biochem. Pharmacol., 35, 1299-1308.

Kimura, Y., Okuda, H., Mori, K., Okuda, T., and Arichi, S. (1984). Effect of extracts of various kinds of tea on lipid metabolic injury in rats fed peroxidized oil. Nippon Eiyo Shokuryo Gakkaishi, 37, 223-
232. (in Japanese).

Kornbrust, D.J. and Mavis, R.D. (1980). The effect of paraquat on microsomal lipid peroxidation in vitro and in vivo. Toxicol. Appl. Pharmacol., 53, 323-332.

Lawrence, R.A. and Burk, R.F. (1976). Glutathione peroxidase activity in selenium-deficient rat liver. Biochem. Biophys. Res. Commun., 71, 952-958.

Lowry, O.H., Rosenbrough, N.J., Farr, A.J. and Randahl, R.J. (1951). Protein measurement with the Folin phenol reagent. J. Biol. Chem., 193, 265-275.

Matkovics, B., Szabó, L., Varga, S.I., Barabas, K. and Berencsi, G. (1980). In vivo effects of paraquat on some oxidation in mice and experiment to support the defense against the poisoning. Dev. Biochem., 11B, 367-380.

Nakagami, T., Nanaumi-Tamura, N., Toyomura, K., Nakamura, T. and Shigehisa, T. (1995). Dietary flavonoids as potential natural biological response modifiers affecting the autoimmune system., $J$. Food Sci., 60, 653-656.

Nakagawa, K. and Miyazawa, T. (1997). Absorption and distribution of tea catechin, (-) -epigallocatechin-3-gallate, in rats. J. Nutr. Sci. Vitaminol., 43, 679-684.

Okushiro, K., Matsumoto, N., Kohri, T., Suzuki, M., Nanjo, F., and Hara, Y. (1996). Absorption of tea catechins into rat portal vein. Biol. Pharm. Bull., 19, 326-329.

Susanne, V., Barbara, T., Davids, A., Gerald, W., Mary, K., Julie, W. and Jose, G. (1996). Inhibitory effect of six green tea catechins and caffeine on the growth of four selected human tumor cell lines. Anti-Cancer Drugs, 7, 461-468.

Toda, H., Okubo, S., Hara, Y. and Shimamura, T. (1991). Antibacterial and bactericidal activities of tea extracts and catechins against methicillin resistant Staphylococcus aureus. Nippon Saikingaku Zasshi, 46, 839-845 (in Japanese).

Tomita, K. and Sano, M. (1983). In "Kasanka Shishitsu Jikkenho," ed. by T. Kaneda, and N. Ueda, Ishiyaku Shuppan Co., Tokyo, pp. 149-150 (in Japanese).

Tsunenari, S., Kibayashi, K. and Furusawa, Y. (1992). Green tea and its effective substance, epigallocatechin gallate $(\mathrm{EGCg})$, reduce paraquat toxicity in mice. Ho Chudoku, 10, 84-85 (in Japanese).

Uchiyama, M. and Mihara, M. (1978). Determination of malonaldehyde precursor in tissue by thiobarbituric acid test. Anal. Biochem., 86, 271-278.

Yagi, K. (1976). A simple fluorometric assay for lipoperoxide in blood plasma. Biochem. Med., 15, 212-216.

Yen, G.-C. and Chen, H.-Y. (1995). Antioxidative activity of various tea extracts in relation to their antimutagenecity. J. Agric. Food Chem., 43, 27-32

Yoshino, K., Tomita, I., Sano, M., Oguni, I., Hara, Y. and Nakano, M. (1994). Effects of long-term dietary supplement of tea polyphenols on lipid peroxide levels in rats. Age, 17, 79-85.

Yoshizawa, S., Horiuchi, T. and Fujiki, H. (1987). Antitumor promoting activity of (-) -epigallocatechin gallate, the main constituent of "Tannin" in green tea. Phytother. Res., 1, 44-47. 